\title{
De l'émigration interne à l'émigration internationale : Impact d'une stratégie de survie sur la pauvreté et les inégalités de revenu au Sénégal
}

\author{
TAMO MBOUYOU Eric Stève ${ }^{1} \&$ KAMGAING SIMO Floriane ${ }^{2}$ \\ ${ }^{1}$ Chercheur associé à la Cellule d'Appui à la Recherche et à l'Enseignement des Institutions \\ Francophones d'Afrique (CARE-IFA/UIESP) \\ Cameroun \\ tamoerics@gmail.com \\ ${ }^{2}$ Doctorante en démographie à l'Université de Montréal \\ Canada \\ florianekamgaing@gmail.com
}

\section{Résumé}

Cet article mesure l'impact des envois de fonds des migrants internes et internationaux sur la pauvreté et les inégalités de revenu, à l'aide des données de l'« Enquête migration et transferts de fonds au Sénégal " réalisée par le Consortium pour la Recherche Economique et Sociale avec le soutien de la Banque mondiale. En considérant les envois de fonds comme étant des substituts potentiels des gains produits localement par le ménage en absence de migration, il ressort des analyses que ces fonds améliorent significativement le bien-être des bénéficiaires, grâce notamment aux gains de consommation qu'ils génèrent. Cependant, seuls les transferts internationaux permettent une baisse significative du taux de pauvreté, au contraire des transferts internes qui eux n'ont pas d'impact. En outre, comme seuls les ménages les plus aisés bénéficient le plus de ces deux types de transferts, cela a pour conséquence un renforcement des inégalités.

Keywords: Migration, Envois de fonds, Pauvreté, Inégalités, Sénégal

\section{Abstract}

This paper measures the impact of remittances from the domestic and international migrant on poverty and inequality, using data from "Senegal - Migration and Remittances Household Survey" conducted in 2009 by the Consortium for Social and Economic Research with support from the World Bank. Considering remittances as potential substitutes gains locally by the household in the absence of migration, it analyzes showed that these funds significantly improve the well-being of beneficiaries. However, only international remittances reduce the incidence of poverty, instead the internal remittances have no impact. In addition, only the wealthiest households benefit most from these two types of remittances. This has resulted in greater inequality.

Keywords: Migration, Remittances, Poverty, Inequality, Senegal

\section{Introduction}

L'émigration interne et l'exode rural en particulier sont deux phénomènes migratoires ayant marqué la plupart des pays en développement notamment d'Afrique subsaharienne, dès l'orée des indépendances. L'essor économique qu'ont connu ces pays à partir des années 1960 s'est accompagné d'une redistribution spatiale de la population avec un exode croissant des campagnes vers les villes du fait de leur plus grande attractivité économique. Les données de recensements et d'enquêtes réalisés depuis lors vont ainsi révéler une urbanisation galopante à l'échelle du continent.
Présentés à l'origine comme une tradition initiatique symbolisant dans les sociétés africaines le passage de la jeunesse à l'âge adulte (Sindjoun, 2004 ; Ela et Zoa, 2006), l'émigration va s'établir progressivement comme une véritable stratégie de survie tant pour les migrants eux-mêmes que pour leur famille d'origine, face aux incertitudes. Ceci va d'ailleurs justifier, au plan scientifique, la prédominance des approches économiques dans l'explication de ce phénomène par rapport aux autres disciplines: sociologie, géographique, démographie, anthropologie, etc.

Cependant, les espoirs soulevés par ces déplacements des campagnes vers les villes vont, en 
l'espace de quelques décennies, être pour la plupart déçusi. Et pour cause, de moins en moins de possibilités seront offertes dans ces lieux de destination, réduisant ainsi les chances d'un épanouissement socioéconomique véritable. Dès lors, l'émigration internationale va s'imposer de plus en plus aux yeux de ces immigrés, mais aussi des " natifs ", comme la nouvelle stratégie de survie : d'où l'accroissement des flux migratoires, mais aussi l'intensification des transferts matériels et immatériels observés entre pays d'origine et de destination.

Cette migration internationale va ainsi connaître, au plan politique notamment, un regain d'intérêt qui va se traduire par la tenue successive de plusieurs conférences internationales dans le cadre du Dialogue de haut niveau sur les migrations internationales et le développement, dont celui de 2013 permettra de souligner non seulement la contribution importante des migrations à la réalisation des Objectifs du Millénaire pour le Développement, mais également de reconnaitre la mobilité humaine comme un facteur décisif du développement durable. Cette contribution positive des migrants sera d'ailleurs reconnue, dans le cadre de l'Agenda de développement pour l'après2015, comme l'un des "catalyseurs" de ce processus.

II importe de souligner cependant qu'un tel intérêt intervient dans un contexte marqué par la mise en œuvre de politiques sélectives dans la majorité des pays du Nord, lesquelles se justifieraient bien par le ralentissement de la croissance économique observé ici, la crise de l'emploi, l'afflux de réfugiés et même par la montée du terrorisme transfrontalier. D'où une circonscription des débats sur ce phénomène migratoire autour de la régulation des flux aux frontières. Or il va sans dire que tout ceci ne saurait, à première vue, être sans conséquences sur le développement des pays d'origine quand on sait que ces mouvements migratoires s'accompagnent habituellement d'une intensification des transferts matériels et immatériels.

Et pourtant, en dépit de ces politiques sélectives et la multiplication des expulsions dans certains pays d'accueil, les envois de fonds des migrants demeurent l'une des principales sources de ressources extérieures pour nombre de pays en développement. En 2015, les fonds transférés par les migrants à destination de leur pays d'origine s'élevaient à 581,6 milliards de dollars dont 431,6 milliards (soit 74\%) à destination des pays en développement (Banque mondiale, 2016). L'émigration internationale seraitelle, au regard de l'importance des sommes en jeu, la meilleure stratégie pour ces migrants et leur famille restée au pays, en comparaison à l'émigration interne?
Si les envois de fonds (internes ou internationaux) des migrants sont dans la plupart des cas sous-tendus par les mêmes motifs et constituent une source de revenu additionnel importante pour de nombreuses familles, l'émigration, qu'elle soit interne ou internationale, ne concerne pas forcément des populations aux caractéristiques similaires, et les dividendes qui en résultent ne sauraient par conséquent être identiques. En effet, non pas d'avoir uniquement des trajectoires différentes, ces deux phénomènes ne mobilisent pas, à priori, les mêmes ressources et encore moins les mêmes réseaux, indispensables pour l'accueil, l'insertion résidentielle et économique des migrants.

De même, la question des transferts monétaires continue de faire l'objet de plusieurs controverses dans le débat relatif aux coûts et bénéfices de la migration. D'un côté, on peut argumenter que les fonds transférés ont un impact positif en constituant une importante source de revenu additionnel pour de nombreux ménages. En revanche, et partant du fait que les fonds reçus sont généralement affectés aux besoins de consommation courante plutôt qu'à des fins productives, on peut opposer à cet argument celui selon lequel ceux-ci placent les bénéficiaires dans une situation de dépendance. La présente recherche a donc pour objectif d'une part, de dresser le profil des ménages récipiendaires des envois de fonds internes et internationaux et, d'autre part, d'évaluer suivant une démarche comparative, la contribution des migrations internes et internationales au développement des ménages d'origine, notamment à travers la mesure de l'impact des envois de fonds sur la pauvreté et les inégalités de revenu. Pour ce faire, les données de l'Enquête migration et transferts de fonds au Sénégal réalisée en 2009 par le Consortium pour la Recherche Economique et Sociale avec le soutien de la Banque mondiale sont utilisées.

Ce travail s'articule autour de trois points. Le premier présente un bref aperçu empirique de l'incidence des envois de fonds sur le bien-être des bénéficiaires. Le deuxième expose le cadre méthodologique. Enfin, le troisième est une synthèse des résultats obtenus.

\section{Impact des transferts de fonds sur le bien-être des} ménages d'origine

En l'absence d'un système de protection sociale et d'assurance, les transferts de fonds constituent une source de revenu additionnel importante pour de nombreuses familles. Ils permettent l'accès aux services sociaux de base comme l'éducation et la santé (Boutin, 201I ; Lopez-Cordova, 2006 ; Franck et Hummer, 2002). En ce qui concerne particulièrement l'impact sur la pauvreté, Adams et 
Page (2005, 2003), Anyanwu et Erhijakpor (2010) démontrent à partir de données sur des pays en développement qu'une hausse des transferts des migrants entraine une baisse du pourcentage d'individus vivant sous le seuil de pauvreté, soit un dollar par jour.

En considérant les envois de fonds comme des substituts potentiels des gains générés localement par le ménage, c'est-à-dire en l'absence de migration, Gubert et al. (2010) établissent que les transferts de fonds entrainent une réduction de la pauvreté au Mali. Utilisant le même scénario contrefactuel que précédemment, des résultats similaires ont été obtenus dans plusieurs pays d'Afrique, notamment au Ghana (Adams et al., 2008) et en Égypte (Adams, 199|).

Si l'impact positif des transferts de fonds sur la pauvreté semble être une évidence parmi les bénéficiaires, celui sur les inégalités de revenu est cependant plus difficile à établir. II n'est pas du tout possible d'affirmer avec certitude que les transferts de fonds permettent une convergence ou une divergence de revenu par rapport à la situation initiale, compte tenu de la diversité des contextes étudiés du point de vue des inégalités initiales, de celle des modèles migratoires et des disparités de résultats dues aux différences de méthode empirique appliquée (Wanner, 2008 ; Efionayi-Mäder et al., 2008 ; Gubert, 2005). Si l'on peut observer au Mali (Gubert et al., 20l0) de même qu'au Mexique (Taylor et al., 2005) une baisse de ces inégalités en présence des transferts internationaux, ceci n'est pas le cas du Ghana (Adams et al., 2008) ni de l'Égypte (Adams, 1991) et encore moins du Nicaragua (Barham et Boucher, 1998) où elles se renforcent et sont au cœur des dysfonctionnements sociaux. La principale explication tient ici au fait que les familles les mieux nanties, contrairement aux plus démunies, participent davantage à l'émigration internationale, car plus à même de supporter les coûts y afférents et bénéficient donc en retour plus largement de ses retombées.

\section{Aspects méthodologiques}

Après avoir présenté la source de données puis défini les principaux concepts utilisés, nous exposerons en fin la méthode d'analyse mobilisée pour cette étude.

\section{Source de données}

Nos travaux mobilisent les données de l'" Enquête migration et transferts de fonds au Sénégal " réalisée en 2009 par le Consortium pour la Recherche Economique et Sociale (CRES) avec le soutien de la Banque mondiale. L'enquête a portée sur 1953 ménages répartis sur l'ensemble du territoire national. La sélection des ménages à enquêter a été http://aps.journals.ac.za faite suivant un échantillonnage aléatoire à deux degrés : Au premier degré, 100 Districts de Recensement (DR) ont été tirés en distinguant les $D R$ à forte migration $(25 \%$ ou plus de ménages avec migrants) de ceux à faible migration (moins de $25 \%$ de ménages avec migrants). Au final, $70 \%$ des DR à forte migration et $30 \%$ des DR à faible migration ont été retenus à l'intérieur de trois strates : Dakar Urbain, Autre Urbain et Rural. Les quotas suivants ont été fixés : 30 DR dans Dakar urbain, 35 dans les autres zones urbaines et 35 dans les zones rurales.

Au deuxième degré, 21 ménages répartis en trois catégories : sans migrant (7), avec migrants internes (7), avec migrants internationaux ou mixtes (migrant interne et internationaux) (7) ont été tirés de façon aléatoire dans chacun des 100 DR retenus.

\section{Définition des concepts}

i) Les transferts de fonds sont des échanges interindividuels mettant en relation deux entités géographiques, en l'occurrence deux pays (transferts internationaux) ou deux localités d'un même pays (transferts nationaux). Ils désignent les biens en numéraire envoyés par le migrant à destination de son ménage d'origine. Dans le cadre de cette étude, seuls les transferts en provenance d'un ancien membre du ménage sont pris en compte.

La mesure des transferts de fonds portait sur une période donnée : les douze mois précédant l'enquête et concernait les ménages ayant connu le départ pour l'étranger d'au moins un de leurs anciens membres, définis comme des personnes qui ont vécu dans le ménage, mais qui à la date de l'enquête vivent à l'extérieur du pays (émigrants internationaux) ou dans une autre région du pays (émigrants internes) depuis au moins une année. Sont également considérées comme anciens membres, des personnes qui ont vécu dans le ménage mais qui à la date de l'enquête l'ont quitté depuis moins d'une année avec l'intention de résider à l'extérieur du pays ou de la région.

Les ménages bénéficiaires désignent ceux avec au moins un ancien membre et qui ont reçu de ce dernier au moins un transfert monétaire au cours des 12 derniers mois précédant l'enquête. Nous distinguons dans la suite de nos travaux deux types de bénéficiaires : internes et internationaux. La première catégorie renvoie aux ménages ayant au moment de l'enquête des émigrants internes exclusivement, tandis que la deuxième fait référence aux ménages avec des émigrants internationaux uniquement.

Les ménages non bénéficiaires représentent ceux sans émigrants (internes ou internationaux) et qui, par ailleurs, n'ont pas reçu d'une tierce personne (parents, amis, etc.) résidant hors du Sénégal le 
moindre transfert monétaire au cours des 12 derniers mois précédant l'enquête.

ii) Pour mesurer la pauvreté, nous recourons dans cet article à l'approche monétaire. Celle-ci présente l'avantage de refléter la photographie de la société à un moment précis donné. En effet, les conditions d'existence utilisées dans l'approche non monétaire s'améliorent moins vite dans le temps, voire quasiment plus au-delà d'un certain standing, et sont par conséquent difficilement rattachables à l'émigration. Au Sénégal, les biens possédés par le ménage de même que les caractéristiques de son habitat ont de fortes chances d'être identiques avant mais aussi après le départ du migrant, dans la mesure où les fonds reçus de ce dernier sont prioritairement destinés à la satisfaction des besoins de consommation courants notamment la santé et l'éducation quel que soit le type d'émigration, comme illustre le graphique I.

Graphique I : Dépenses moyennes effectuées à partir des transferts monétaires reçus selon le poste d'affectation et le statut migratoire du ménage

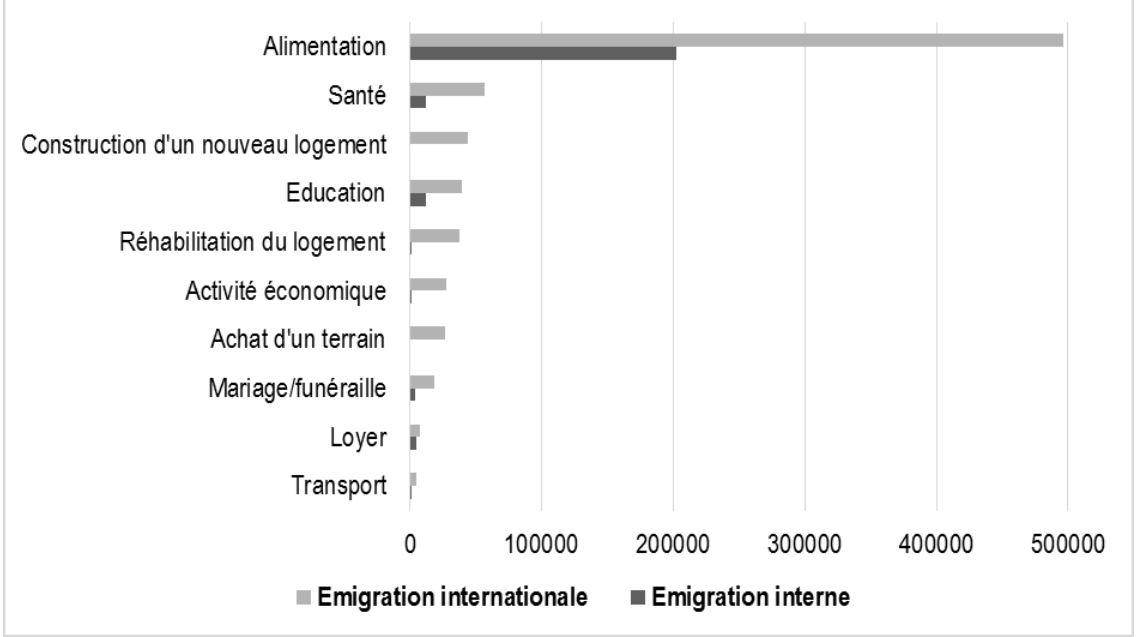

Source : Calculs des auteurs à partir des données CRES 2009

La mesure de l'impact des transferts fonds des migrants porte sur les indicateurs suivants : (i) la dépense par tête, (ii) l'incidence, la profondeur et la sévérité de la pauvreté et (iii) l'indice de Gini.

Indicateur de bien-être monétaire, la dépense par tête (proxy du revenu par tête) désigne la dépense annuelle qu'effectue en moyenne un ménage pour chacun de ses membres. Elle prend en compte tous les besoins aussi bien biologiques (nutrition) que sociaux (santé, éducation, logement, habillement, transport, etc.) pouvant faire l'objet d'une valorisation monétaire. En ce qui concerne l'incidence de la pauvreté, elle se définit comme la proportion d'individus vivant sous le seuil de pauvreté. En 2010, l'Agence Nationale de la Statistique et de la Démographie du Sénégal (20/3) fixait ce seuil à I 053 FCFA par personne et par an dans la ville de Dakar, 798 FCFA dans les autres zones urbaines et 598 FCFA en zone rurale. Sur la base de ces lignes de pauvreté, l'incidence de la pauvreté (ou taux de pauvreté) était évalué à $46,7 \%$ à l'échelle du pays dont $26,1 \%$ à Dakar, $41,2 \%$ dans les autres zones urbaines et $57,1 \%$ en milieu rural. Afin d'avoir approximativement ces mêmes taux de pauvreté au sein de l'échantillon total de l'enquête CRES de 2009, nous retiendrons comme seuils de pauvreté 653,443 et 366 FCFA respectivement à 3813
Dakar, dans les autres localités urbaines et dans les zones rurales.

S'agissant de la profondeur de la pauvreté, elle mesure la distance moyenne qui sépare les pauvres du seuil de pauvreté. La sévérité de la pauvreté mesure quant à elle la différence d'intensité (inégalité) de la pauvreté parmi les pauvres. Pour ce qui est de l'indice de Gini, il est utilisé pour la mesure des inégalités de revenu et est compris entre 0 et $\mathrm{I}$. Plus il est proche de I, plus l'inégalité mesurée est importante.

Ces trois indicateurs se calculent à l'aide de la formule suivante :

$$
P_{\alpha}=\frac{1}{n} \sum_{i=1}^{n}\left(\frac{Z-Y_{i}}{Z}\right)^{\alpha} \varepsilon
$$

- $\quad Y_{i}$ représente la dépense par tête du ménage $i$ et $Z$ le seuil de pauvreté

$\varepsilon$ est une fonction indicatrice qui prend la valeur I si $Z>Y_{i}$ et 0 sinon.

Pour :

- $\alpha=0, P_{0}$ désigne l'incidence de la pauvreté ;

- $\alpha=1, P_{1}$ désigne la profondeur de la pauvreté ;

- $\quad \alpha=2, P_{2}$ désigne la sévérité de la paurreté. 
Tableau I : Indicateurs de pauvreté au Sénégal

\begin{tabular}{|c|c|c|c|c|}
\hline Indicateur de pauvreté & Dakar & $\begin{array}{l}\text { Autre zone } \\
\text { urbaine }\end{array}$ & $\begin{array}{l}\text { Zone } \\
\text { rurale }\end{array}$ & Ensemble \\
\hline Seuil de pauvreté de l'ESPS-II 20I0/20II & I 053 & 798 & 598 & - \\
\hline Taux de pauvreté de l'ESPS-II 2010/20II & 26,1 & 41,2 & 57,1 & 46,7 \\
\hline Profondeur de la pauvreté de l'ESPS-II 2010/201। & 5,8 & 13,1 & 18,6 & 14,5 \\
\hline Sévérité de la pauvreté de l'ESPS-II 2010/201I & 2,1 & 5,9 & 6,7 & 6,1 \\
\hline Seuil de pauvreté retenu pour l'échantillon CRES 2009 & 653 & 443 & 366 & - \\
\hline Taux de pauvreté au sein de l'échantillon CRES 2009 & 26,1 & $4 I, 0$ & 57,0 & 42,2 \\
\hline Profondeur de la pauvreté au sein de l'échantillon CRES 2009 & 8,9 & 13,5 & 23,2 & 15,5 \\
\hline Sévérité de la pauvreté au sein de l'échantillon CRES 2009 & 4,2 & 6,2 & 12,6 & 7,2 \\
\hline
\end{tabular}

Source : ESPS-II, ANSD 2013 et calculs des auteurs à partir des données CRES 2009

\section{Méthodes d'analyse}

Afin de savoir si les transferts de fonds soulagent ou non la pauvreté, il est indispensable de suivre les trajectoires économiques mais aussi migratoires des bénéficiaires, ou du moins de disposer d'une séquence d'observations. Or les panels de ménages perdent justement ceux qui migrent et les enquêtes biographiques rétrospectives, qui restent rares et lourdes, peinent à obtenir une appréciation objective des pauvretés passées (Delaunay, 2006).

Compte tenu donc du caractère transversal de nos données, nous comparons la situation observée, c'est-à-dire qui tient compte des transferts, à un scénario contrefactuel où le migrant resterait et travaillerait dans son ménage d'origine. A l'aide de la méthode des Moindres Carrés Ordinaires (MCO), il s'agit précisément d'estimer le revenu des ménages non bénéficiaires et à utiliser les coefficients estimés pour prédire celui des ménages bénéficiaires en absence de migration. La série ainsi obtenue est comparée aux revenus observés et l'écart qui en résulte est considéré comme la contribution des transferts fonds au bien-être du ménage.

Cependant, une telle approche sous-tend implicitement que les fonds reçus par le ménage ont un impact sur son revenu actuel. Or un tel impact peut résulter des effets directs et/ou indirects des transferts antérieurs sur d'autres sources de revenus : d'où sa principale limite. Suivant cette approche, le revenu contrefactuel des ménages bénéficiaires s'obtient à partir de l'équation suivante :

$$
\log Y_{i}=\alpha+\beta \log H_{i}+\gamma X_{i}+\varepsilon_{i}(I)
$$

Où $Y_{i}$ est une mesure du revenu du ménage bénéficiaire $\mathrm{i}$. $\mathrm{Hi}$ et $\mathrm{Xi}$ sont respectivement les vecteurs des caractéristiques du ménage et celles du chef de ménage, et $\varepsilon$ i est le terme d'erreur.

La prise en compte de la sélection endogène des ménages migrants, c'est-à-dire qui participent à l'émigration internationale ou interne, est indispensable pour éviter une invalidation de la méthode des MCO. En effet, les ménages migrants sont en général les plus dynamiques. Une estimation de leur revenu par la méthode des MCO serait ainsi sous-évaluée, car ces ménages auront vraisemblablement, du fait de leurs caractéristiques non observables, un revenu supérieur à celui des autres ménages même en absence de migration. $\mathrm{Ce}$ qui conduirait à une surévaluation de l'impact des transferts de fonds. Pour corriger ce biais endogénéité introduit par la sélectivité des ménages migrants, le mécanisme de correction utilisé ici s'inspire de celui de Barham et Boucher (1988). La règle de (non) sélection est modélisée à partir de la spécification probit suivante :

$$
M_{i}=\alpha_{m}+\beta_{m} \log H_{i}+\gamma X_{i}+\delta_{m} Z_{i}+\varepsilon_{i}(2)
$$

Où $M$ vaut $I$ quand le ménage ne participe pas à l'émigration interne (respectivement l'émigration internationale) et 0 sinon.

$\mathrm{Zi}$ est le vecteur de variables explicatives d'une non participation du ménage à l'émigration internationale (respectivement l'émigration interne), mais sans effet sur le revenu de ménage ainsi que les caractéristiques non observables contenues dans $\varepsilon_{\mathrm{i}}$. On fait l'hypothèse qu'un ménage migrant est doté d'une fonction de production générant un revenu $Y_{1}$; dans le cas contraire, sa fonction de production a des coefficients différents et génère un revenu $Y_{0}$.

A partir de l'équation (2), nous estimons la probabilité pour un ménage de ne pas participer à l'émigration internationale (respectivement l'émigration interne) ; probabilité à partir de laquelle on construit l'inverse du Ratio de Mill $(\lambda)$ qui permet de corriger les biais liés à la sélection endogène des ménages migrants. L'équation (I) peut ainsi de nouveau s'écrire :

$$
\begin{aligned}
& \log Y_{\mathbf{i}}=\boldsymbol{\alpha}+\boldsymbol{\beta} \log \mathbf{H}_{\mathbf{i}}+\boldsymbol{\gamma} \mathbf{X}_{\mathbf{i}}+\boldsymbol{\lambda}_{\mathbf{i}}+\boldsymbol{\mu}_{\mathbf{i}} \text { (3) } \\
& \text { Où } \mu \mathrm{i} \text { est le nouveau terme d'erreur, }
\end{aligned}
$$
indépendamment et identiquement distribué. 


\section{Description des variables}

Les termes $\mathrm{Hi}$ et $\mathrm{Xi}$ des équations (I), (2) et (3) sont respectivement les vecteurs des caractéristiques du ménage et celles du chef de ménage. II s'agit précisément, au niveau du ménage : du lieu de résidence, d'actifs possédés, de la taille, du nombre d'individus instruits (ayant un niveau secondaire ou plus) qu'il abrite. Notons que la variable " actifs possédés " est un score de biens détenus par le ménage, construit à l'aide d'une analyse en composante principale $(\mathrm{ACP})$ à partir des biens suivants : téléphone, ordinateur, vélo, moto, voiture, l'électricité, l'eau, etc. Ces actifs forment avec le capital humain, opérationnalisé à travers le nombre d'individus instruits, les facteurs qui déterminent le niveau de production du ménage et par conséquent son revenu. Les caractéristiques du chef de ménage sont, quant à elles, appréhendées à travers l'âge, le sexe et la situation d'activité de celui-ci.

Le vecteur $\mathrm{Zi}$ de variables explicatives d'une non participation du ménage à l'émigration -contenu dans l'équation de (non) sélection ou équation (2)- est saisi par la seule variable "l'ethnie du ménage »".ii. En effet, l'émigration dans le cas du Sénégal s'adosse sur " un tissu complexe de rapports sociaux qui apparaissent sous la forme de circuits d'accueil et/ou d'insertion socioprofessionnelle, résidentielle, de solidarité humaine, de relations privilégiées " (Abdou Salam Fall, 1995, p.258). Malgré une tendance de l'accueil à orientation communautaire à l'arrivée d'immigrants dans une autre localité du pays ou dans un autre pays, les cercles familiaux et amicaux sont fortement mis à contribution, ce qui participe à la construction de réseaux sociaux et amicaux à travers des relations amicales et conjugales diversifiées. Dès lors, la propension du ménage à participer ou non à l'émigration interne ou internationale varierait par exemple selon le groupe ethnique auquel appartient le migrant (Annexe I).

\section{Principaux résultats}

Caractéristiques des transferts monétaires au Sénégal

Avant de procéder à une analyse comparative entre les caractéristiques des ménages bénéficiaires et celles des non récipiendaires des transferts monétaires internes et internationaux, il importe, d'entrée de jeu, de présenter brièvement les caractéristiques des transferts monétaires internationaux au Sénégal sous une perspective macroéconomique.

Entre 2003 et 2010, les fonds transférés par les migrants à destination du Sénégal n'ont pratiquement pas cessé d'augmenter (Graphique 2). Cependant, la croissance quasi stable observée sur plusieurs années a connu en 2009 une légère baisse consécutive à la crise financière mondiale, dont les effets se sont particulièrement fait ressentir dans les principaux pays d'émigration.

Graphique 2 : Évolution des transferts de fonds internationaux à destination du Sénégal (2003-2010)

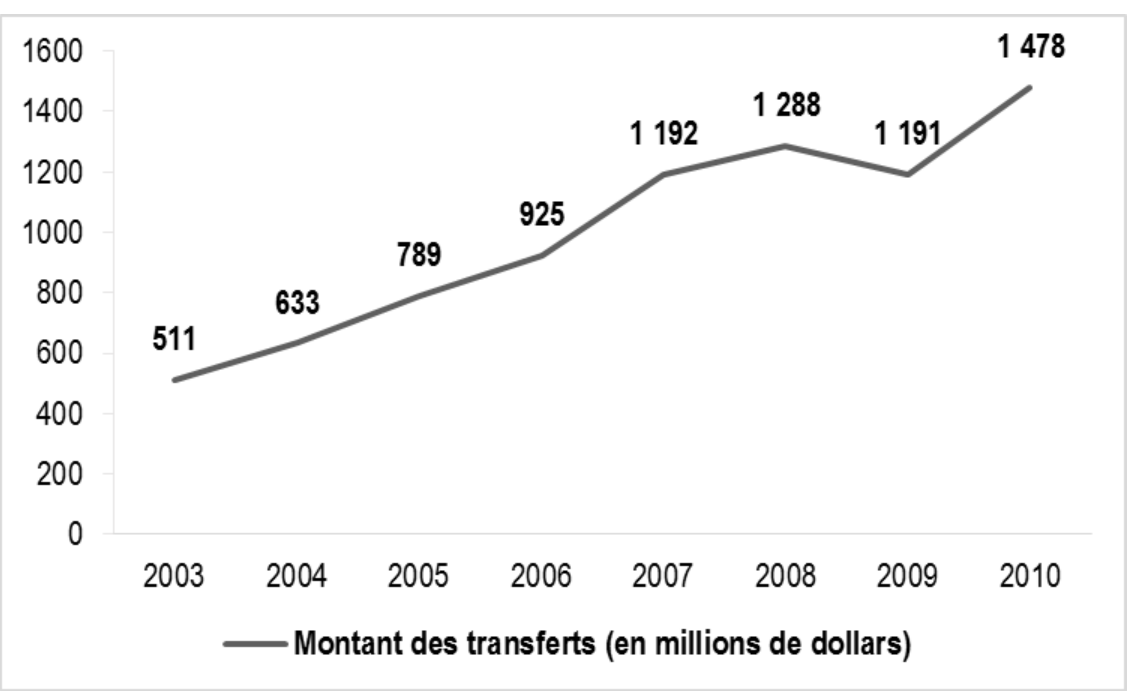

Source : Migration and Remittances Factbook 20II, données du site http://go.worldbank.org/ITC7NYTT0, 2016

Évalués à | 478 millions de dollars en 2010 soit II,4\% du PIB, ces fonds ne sont bien évidemment que l'une des conséquences des migrations. En 2010, la population d'émigrants sénégalais était estimée à 210061 individus répartis majoritairement entre
l'Afrique et l'Europe ${ }^{\mathrm{i} v}$. La France, qui est la quatrième destination de ces émigrants, occupe la première place devant l'Italie en ce qui concerne l'entrée des fonds en provenance de l'étranger (Graphique 3). Ces deux pays sont suivis par l'Espagne puis les Etats 
Unis pour ce qui relève des transferts Nord-Sud ; la Gambie, la Mauritanie, le Gabon et la Côte d'Ivoire étant ces principaux pays d'envoi dans le cadre de transferts Sud-Sud.

Graphique 3: Principaux pays d'origine des fonds transférés au Sénégal en 2010

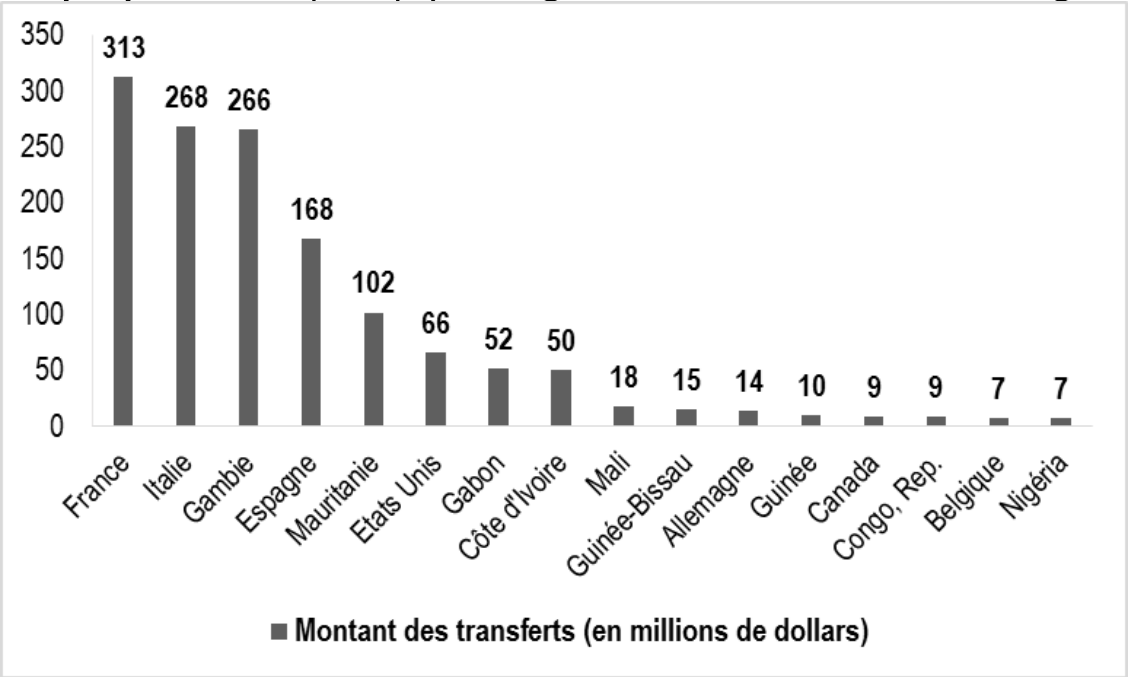

Source : Données du site http://go.worldbank.org/ITC7NYTT0, 2016

Les ménages bénéficiaires de transferts monétaires internationaux semblent afficher un niveau de bienêtre monétaire de loin supérieur à celui des ménages sans émigrants, mais également à celui des ménages récipiendaires de transferts internes. En effet, un ménage qui reçoit exclusivement des transferts internationaux dépense en moyenne 405049 FCFA par membre et par an, contre 234959 FCFA pour un ménage percevant uniquement des transferts internes et 388029 FCFA pour un ménage sans émigrants. Ceci pourrait s'expliquer par le fait que les sommes reçues par les ménages avec émigrants internationaux sont en moyenne près de trois fois supérieur à celles perçues par des ménages avec émigrants internes. Les premiers reçoivent en moyenne 814 136 FCFA contre 264617 FCFA pour les deuxièmes. En outre, l'analyse des caractéristiques migratoires du ménage nous révèle qu'en moyenne, le nombre d'émigrants au départ des ménages bénéficiaires de transferts internationaux $(I, 6)$ est quasiment égal à celui-ci des ménages qui reçoivent des transferts internes $(1,52)$. A ce stade, de tels résultats supposeraient qu'une émigration internationale serait bien plus profitable pour le ménage qu'une simple émigration interne.

Les ménages se localisant dans la ville de Dakar bénéficient le plus de transferts internationaux tandis que ceux des autres localités urbaines et des zones rurales occupent les premières places pour ce qui est transferts internes. Au plan culturel, les ménages originaires de l'ethnie Pular bénéficient particulièrement de transferts monétaires tant internes qu'internationaux, beaucoup plus que les autres ethnies.

La taille du ménage aurait un effet important sur la réception de fonds. II en est de même du nombre de personnes de la tranche d'âge inactive au sein du ménage. En effet, un ménage de grande taille est synonyme de besoins, tout au moins en termes d'alimentation, plus importants. Les chances pour un ménage sénégalais de bénéficier de transferts soit internes soit internationaux seraient d'autant plus élevées que la taille du ménage est grande, et probablement plus encore lorsque le nombre d'individus à charge (dépendants) qu'il abrite est important. En effet, la taille moyenne d'un ménage ne bénéficiant pas de transfert est la plus faible $(7,81)$. En revanche, elle est de 9,1 parmi les bénéficiaires de transferts internes et de 10,05 individus chez ceux recevant des transferts internationaux.

D'après les résultats de la deuxième Enquête de Suivi de la Pauvreté au Sénégal (ESPS II), la pauvreté (monétaire) est moins répandue dans les foyers dirigés par des femmes que dans ceux dirigés par des hommes : $34,7 \%$ des personnes vivant dans des ménages dirigés par des femmes sont en situation de pauvreté contre $50,6 \%$ chez les personnes qui vivent dans les ménages dirigés par les hommes (ANSD, 20I3). Un tel résultat se justifierait bien par le fait qu'une frange importante de ces ménages bénéficiant de transferts monétaires internes a à sa tête des femmes (33\%). II en est de même des ménages qui perçoivent des transferts internationaux (4I\%). 
Tableau 2 : Description des ménages bénéficiaires et non récipiendaires de transferts

\begin{tabular}{|c|c|c|c|c|c|c|}
\hline \multirow[t]{2}{*}{ Type de ménage } & \multicolumn{2}{|c|}{$\begin{array}{l}\text { Ménages sans émigrants } \\
(n=657)\end{array}$} & \multicolumn{2}{|c|}{$\begin{array}{l}\begin{array}{l}\text { Ménages bénéficiaires avec émigrants } \\
\text { internes exclusivement } \\
(n=442)\end{array} \\
\end{array}$} & \multicolumn{2}{|c|}{$\begin{array}{l}\text { Ménages bénéficiaires avec émigrants } \\
\text { internationaux exclusivement } \\
(\mathrm{n}=534)\end{array}$} \\
\hline & Moyenne & Ecart-type & Moyenne & Ecart-type & Moyenne & Ecart-type \\
\hline Dépenses moyenne par personne et par an & 388029 & 652801 & 234959 & 266188 & 405049 & 768358 \\
\hline Montant moyen reçu par ménage & 0 & 0 & 264617 & 460986 & $814 \mid 36$ & $|323| 28$ \\
\hline \multicolumn{7}{|l|}{ Lieu de résidence du ménage : } \\
\hline Dakar* & 0,32 & 0,47 & 0,17 & 0,37 & 0,37 & 0,48 \\
\hline Autre zone urbain* & 0,37 & 0,48 & 0,45 & 0,50 & 0,33 & 0,47 \\
\hline Rural* & 0,31 & 0,46 & 0,39 & 0,49 & 0,30 & 0,46 \\
\hline \multicolumn{7}{|l|}{ Ethnie du ménage } \\
\hline Bambara* & 0,02 & 0,12 & 0,02 & 0,12 & 0,02 & 0,14 \\
\hline Diola* & 0,03 & 0,18 & 0,05 & 0,23 & 0,02 & 0,14 \\
\hline Pular* & 0,33 & 0,47 & 0,34 & 0,47 & 0,36 & 0,48 \\
\hline Sérer* & 0,10 & 0,30 & 0,10 & 0,30 & 0,06 & 0,23 \\
\hline Autres* & 0,10 & 0,30 & 0,05 & 0,23 & 0,08 & 0,28 \\
\hline Scores d'actifs possédés par le ménage & $-0,21$ & 1,97 & $-0,48$ & 1,68 & 0,61 & 2,10 \\
\hline Taille du ménage & 7,85 & 4,68 & 9,10 & 4,80 & 10,05 & 6,13 \\
\hline Nombre de dépendants dans le ménage & 3,26 & 2,84 & 4,03 & 3,10 & 4,32 & 3,63 \\
\hline Nombre d'instruits dans le ménage & I,34 & I,95 & 1,36 & 1,95 & I,93 & 2,44 \\
\hline $\begin{array}{l}\text { Nombre de personnes économiquement occupées } \\
\text { dans le ménage }\end{array}$ & 2,45 & 1,95 & 2,58 & 1,92 & 2,48 & 2,31 \\
\hline Nombre d'émigrants internes du ménage & 0,00 & 0,00 & 1,52 & 0,93 & 0,00 & 0,00 \\
\hline Nombre d'émigrants internationaux du ménage & 0,00 & 0,00 & 0,00 & 0,00 & 1,60 & 0,99 \\
\hline Femme chef de ménage* & 0,17 & 0,38 & 0,33 & 0,47 & 0,41 & 0,49 \\
\hline Age du chef de ménage & 50,91 & 14,49 & 52,54 & 14,22 & 53,64 & 15,18 \\
\hline Age au carré du chef de ménage & $280 I, 41$ & 1567,90 & 2961,86 & 1523,24 & $3107,8 \mid$ & $\mid 691,05$ \\
\hline Chef de ménage économiquement occupé* & 0,75 & 0,43 & 0,69 & 0,46 & 0,59 & 0,49 \\
\hline
\end{tabular}

* Variable muette

Source : Calculs des auteurs à partir des données CRES 2009 
Impact des transferts de fonds sur la pauvreté

L'estimation du revenu des ménages non bénéficiaires nous permet d'obtenir, en tenant compte des biais liés à la sélection des ménages non migrants (internes et internationaux), les coefficients servant à prédire celui des ménages récipiendaires en l'absence de migration. Dans l'ensemble, les signes de ces coefficients sont conformes aux attentes théoriques (Tableau 3).

Le revenu du ménage varie en fonction du lieu de résidence. Les ménages résidant dans la ville de Dakar ont un revenu supérieur à celui des ménages se localisant dans les autres zones urbaines du pays. La taille du ménage a un effet négatif sur le revenu. Autrement dit, le bien-être du ménage diminue avec une augmentation du nombre d'individus en son sein. En outre, on constate une relation croissante entre le revenu du ménage et le nombre de personnes instruites qu'il abrite.

En plus d'être significatif, l'inverse du Ratio de Mill (Lambda) dans le cas d'une prise en compte des biais liés à la sélection des ménages non migrants internationaux a un signe positif, ce qui signifie qu'il existe une corrélation positive entre d'une part, le terme d'erreur de l'équation de (non) sélection et, d'autre part, le terme d'erreur de l'équation de revenu, validant ainsi la méthode d'estimation utilisée. II en est de même, dans une moindre mesure cette fois, de la prise en compte des biais liés à la sélection des ménages non migrants internes; quoique la corrélation entre le terme d'erreur de l'équation de (non) sélection et le terme d'erreur de l'équation de revenu soit plutôt ici négative.

Tableau 3 : Estimation du revenu des ménages sans migrant

\begin{tabular}{|c|c|c|c|c|c|c|c|}
\hline \multirow{2}{*}{$\begin{array}{l}\text { Type d'émigration } \\
\text { Caractéristique }\end{array}$} & \multicolumn{3}{|c|}{ Emigration interne } & \multicolumn{4}{|c|}{ Emigration internationale } \\
\hline & $\begin{array}{l}\text { Sans } \\
\text { correction }\end{array}$ & \multicolumn{2}{|c|}{$\begin{array}{l}\text { Avec } \\
\text { correction }\end{array}$} & \multicolumn{2}{|c|}{$\begin{array}{l}\text { Sans } \\
\text { correction }\end{array}$} & \multicolumn{2}{|c|}{$\begin{array}{l}\text { Avec } \\
\text { correction }\end{array}$} \\
\hline \multicolumn{8}{|l|}{ Milieu } \\
\hline Dakar & Réf. & Réf. & & Réf. & & Réf. & \\
\hline Autre urbain & $-0,18$ & 0,03 & & $-0,18$ & & 0,07 & \\
\hline Rural & $-0,87 * * * *$ & $-0,62$ & **** & $-0,87$ & ***** & $-0,74$ & **** \\
\hline Taille du ménage (Log) & $-0,61 * * * *$ & $-0,61$ & **** & $-0,61$ & $* * *$ & $-0,79$ & *** \\
\hline Nombre d'instruits (Log) & $0,22 * *$ & 0,21 & ** & 0,32 & ** & 0,08 & \\
\hline $\begin{array}{l}\text { Sexe du Chef de ménage } \\
\quad(0=\text { Homme, } I=\text { Femme })\end{array}$ & 0,17 & 0,26 & ** & 0,18 & & $-0,16$ & \\
\hline Age du Chef de ménage & $-0,00$ & 0,00 & & $-0,00$ & & 0,00 & \\
\hline Age du chef de ménage au carré & $7 e-06$ & 0,00 & & $7 e-06$ & & 0,00 & \\
\hline $\begin{array}{l}\text { Situation d'activité du CM } \\
\text { (0=Non occupé, I = Occupé })\end{array}$ & 0,07 & 0,04 & & 0,07 & & 0,12 & \\
\hline Constance & $13,67 * * * *$ & 13,43 & ***** & 13,67 & ***** & 13,57 & **** \\
\hline Inverse du ratio de Mill (Lambda) & $\mathrm{Na}$ & $-0,79$ & $*$ & $\mathrm{Na}$ & & I,44 & **** \\
\hline$R^{2}$ & 53,63 & 54,32 & & 53,63 & & 56,43 & \\
\hline
\end{tabular}

Variable dépendante : Log (Dépenses par tête) avec Log = Logarithme

$\mathrm{Na}:$ Non applicable

*** $\mathrm{p}<0,01,{ }^{* *} \mathrm{p}<0,05,{ }^{*} \mathrm{p}<0,1$

Source : Calculs des auteurs à partir des données CRES 2009

Les transferts de fonds qu'ils soient internes ou internationaux améliorent significativement le bienêtre des bénéficiaires, car les gains de consommation qu'ils génèrent sont très largement positifs (Tableau 4). Cependant, seuls les transferts internationaux permettent une baisse de $15,5 \%$ du taux de pauvreté qui passe de $41 \%$ à $34,6 \%$, quoique l'impact sur la profondeur et la sévérité de la pauvreté ne soit pas significatif. En revanche, l'apport des transferts internes n'a pas incidence significative : la baisse du taux de pauvreté étant ici de $0,4 \%$ seulement. Toutefois, le départ d'un membre du ménage pour une autre localité du pays aggrave la profondeur de la pauvreté, c'est-à-dire la distance entre les pauvres et le seuil de pauvreté.

En outre, les fonds reçus n'entrainent pas un nivelage (une égalisation) des revenus parmi les pauvres. On observe au contraire une hausse des inégalités de revenu de $37,9 \%$ au sein de ce sousgroupe ; certains en bénéficiant plus que d'autres. 
Tableau 4 : Impact des transferts de fonds sur la pauvreté

\begin{tabular}{llllc}
\hline Type d'émigration & Pauvreté & Sans transferts & Avec transferts & Écart (\%) \\
\hline Emigration interne & Dépense par tête & 186389 & 234959 & $26,1 * * *$ \\
& Incidence & 50,79 & 50,57 & $-0,4$ \\
& Profondeur & 14,63 & 17,92 & $22,5 * * *$ \\
& Sévérité & 6,01 & 8,29 & $37,9 * * *$ \\
\hline Emigration internationale & Dépense par tête & $227 \mid 30$ & 405049 & $78,3 * * *$ \\
& Incidence & 41,01 & 34,64 & $-15,5 * * *$ \\
& Profondeur & 13,62 & 12,55 & $-7,9$ \\
& Sévérité & 6,07 & 6,11 & 0,7 \\
\hline$* * *$ & & &
\end{tabular}

Source : Calculs des auteurs à partir des données CRES 2009

Impact des transferts de fonds sur inégalités de revenu

En ce qui concerne l'impact des transferts de fonds sur les inégalités de revenu, on constate que leur présence tend à les renforcer, quel que soit le type de transferts (Tableau 5). Seuls les ménages non pauvres précisément ceux du $4 \mathrm{e}$ et $5 \mathrm{e}$ quintiles les plus riches en tirent un réel profit. Pour le quintile riche, la migration se traduit donc par une hausse de la consommation moyenne. En d'autres termes, les ménages riches reçoivent plus de leurs émigrants que ce qu'ils auraient gagné si ces derniers étaient restés travailler au Sénégal ou dans leur localité d'origine. Ce qui est loin d'être le cas chez les pauvres.

$\mathrm{Ce}$ résultat se vérifie également au niveau de l'indice de Gini dont la valeur croît de 0,3 I à 0,45 soit une hausse de $45,2 \%$ en présence des transferts internes, et de 0,33 à 0,57 soit une augmentation de $63,6 \%$ en présence cette fois des transferts internationaux, traduisant là une exacerbation des inégalités dans les deux cas. Ces résultats sont similaires à ceux de de Adams et al. (2008) dans le cas du Ghana et de Barham et Boucher (1998) au Nicaragua.

Tableau 5 : Impact des transferts de fonds sur les inégalités de revenu

\begin{tabular}{|c|c|c|c|c|c|}
\hline Type d'émigration & Quintile de bien-être & Sans transferts & Avec transferts & Écart (\%) & \\
\hline \multirow[t]{8}{*}{ Emigration interne } & Pauvre & 146789 & 102189 & $-30,4$ & **** \\
\hline & Non pauvre & 226898 & 370774 & 63,4 & **** \\
\hline & QI & 109294 & 70651 & $-35,4$ & **** \\
\hline & Q2 & 156606 & 126850 & $-19,0$ & *** \\
\hline & Q3 & 193637 & 189754 & $-2,0$ & \\
\hline & Q4 & 237500 & 309512 & 30,3 & **** \\
\hline & Q5 & 321700 & 757606 & 135,5 & *** \\
\hline & Indice de Gini & 0,31 & 0,45 & 45,2 & **** \\
\hline \multirow[t]{8}{*}{ Emigration internationale } & Pauvre & 149188 & 107234 & $-28,1$ & **** \\
\hline & Non pauvre & 268446 & 562917 & 109,7 & **** \\
\hline & QI & 102167 & 64555 & $-36,8$ & *** \\
\hline & Q2 & $152 \mid 88$ & 125125 & $-17,8$ & *** \\
\hline & Q3 & 187749 & 192555 & 2,6 & \\
\hline & Q4 & 271069 & 312700 & 15,4 & *** \\
\hline & Q5 & 344710 & 1091946 & 216,8 & **** \\
\hline & Indice de Gini & 0,33 & 0,54 & 63,6 & **** \\
\hline
\end{tabular}

**** $\mathrm{p}<0,0$ I

Source : Calculs des auteurs à partir des données CRES 2009 


\section{Conclusion}

Cet article avait pour objectif d'évaluer suivant une démarche comparative la contribution des migrations internes et internationales au développement des ménages d'origine, notamment à travers la mesure de l'impact des envois de fonds sur la pauvreté et les inégalités de revenu. À l'aide des données de l'Enquête migration et transferts de fonds au Sénégal réalisée en 2009 par le CRES, nous avons comparé la situation observée, c'est-à-dire en présence des transferts de fonds, à un scénario contrefactuel où le migrant resterait et travaillerait dans sa localité d'origine ou dans son pays d'origine.

Pour ce faire, nous avons recouru à la méthode des Moindres Carrés Ordinaires pour estimer le revenu des ménages non bénéficiaires puis les coefficients estimés ont été utilisés pour prédire celui des ménages bénéficiaires en absence de migration, le tout après avoir corrigé, à partir d'un mécanisme inspiré de celui de Barham et Boucher (1988), le biais endogénéité introduit par la sélectivité des ménages migrants. Cependant, l'approche utilisée comporte une limite à savoir qu'elle sous-tend implicitement l'impact sur le revenu actuel des fonds reçus par le ménage. Or un tel impact peut résulter des effets directs et/ou indirects des transferts antérieurs sur d'autres sources de revenus.

II ressort des analyses que les transferts monétaires de façon générale améliorent significativement le bien-être des bénéficiaires, car les gains de consommation qu'ils génèrent sont très largement positifs. Cependant, seuls les transferts internationaux permettent une baisse significative de $15,5 \%$ du taux de pauvreté, à la différence des transferts internes qui eux n'ont pas d'impact. Au contraire, le départ d'un membre du ménage pour une autre localité du pays aggrave la profondeur et la sévérité de la pauvreté.

Seuls les ménages les plus riches tirent un réel profit aussi bien de l'émigration interne que de l'émigration internationale, étant donné que les fonds reçus en retour de leurs émigrants sont bien audessus de ce que ces derniers auraient gagné s'ils étaient restés travailler au Sénégal ou simplement dans leur localité d'origine. Ce qui est loin d'être le cas chez les pauvres. Cette situation est à l'origine d'une recrudescence des inégalités en termes de revenu, ce qui est traduit par une hausse de l'indice de Gini.

Ces résultats nous livrent deux principaux enseignements pour l'action des pouvoirs publics. Premièrement, les migrations internationales à travers les envois de fonds qui en résultent participent concrètement au développement du pays d'origine, notamment à travers la lutte contre la pauvreté. Ceci suggère donc qu'une incitation des http://aps.journals.ac.za bénéficiaires pour une utilisation des fonds transférés à des fins productives plutôt que de consommation permettrait à terme de lutter encore plus efficacement contre cette pauvreté. Deuxièmement, malgré un effet positif tel que souligné précédemment, les envois de fonds des migrants sont cependant à l'origine de dysfonctionnements sociaux caractérisés par une aggravation des inégalités de revenu au sein de la population : certaines couches sociales, en l'occurrence les plus nanties, bénéficiant plus largement de ces fonds. Dans un tel contexte, une égalisation ou alors un nivelage de revenu entre ces différentes couches passe, entre autres, par des transferts monétaires des pouvoirs publics vers les ménages les plus pauvres à travers des Programmes de Filets Sociaux par exemple. Toutefois, la soutenabilité d'une telle action dans le temps oblige par ailleurs que ces transferts sociaux soient couplés à des projets de développement et autres initiatives visant à réduire la dépendance de ces derniers à l'égard desdits transferts.

\section{Bibliographie}

Abdou Salam Fall (1995) : "Relation à distance des migrants et réseaux d'insertion à Dakar ", in Antoine P. et Diop A., Ville à guichets fermés ?, IFAN / ORSTOM, pp. 257-275.

Agence Nationale de la Statistique et de la Démographie (20/3) : Deuxième Enquête de Suivi de la Pauvreté au Sénégal (ESPS-II 20II), Rapport définitif, I $22 \mathrm{p}$.

Adams Richard (|99|): "The Economic uses and Impact of International Remittances in Rural Egypt", Economic Development and Cultural Change, 39 (4), pp. 695-722.

Adams Richard, Cuecuecha Alfredo and Page John (2008): "The Impact of Remittances on Poverty and Inequality in Ghana", World Bank Policy Research Working Paper 4732, $41 \mathrm{p}$.

Anyanwu John and Erhijakpor Andrew (20I0): "Do International Remittances Affect Poverty in Africa”, African Development Review, 22 (I), pp. 5I-9I.

Barham Bradford and Boucher Stephen (1998): "Migration, remittances, and inequality: estimating the net effects of migration on income distribution", Journal of Development Economics, 55, pp. 307-33I.

Boutin Delphine (20II) : "Envoi de fonds et allocation du temps des enfants au Niger : l'effet indirect des chocs négatifs ", LAREFI Working Paper CRII-EFI/05, Bordeaux, Université Bordeaux IV, $33 \mathrm{p}$.

Chami Ralph, Barajas Adolfo, Cosimano Thomas, Fullenkamp Connel, Gapen Michael and Montiel 
Peter (2008): "Macroeconomic Consequences of Remittances", IMF Occasional Paper 259, 94 p.

Delaunay Daniel (2006) : "Relations entre la pauvreté, la migration et les mobilités : dimensions territorial et contextuelle ", Reunión de Expertos sobre Población y Pobreza en América Latina y el Caribe (14 y 15 de Noviembre 2006, Santiago, Chile), 33 p.

Efionayi-Mäder Denise, Perroulaz Gérard et Schümperli Catherine (2008) : "Migration et développement : les enjeux d'une relation controversée ", Annuaire suisse de politique de développement, 27 (2), pp. II-20.

Frank Reann and Hummer Robert (2002): "The Other Side of the Paradox: The Risk of Low Birth Weight among Infants of Migrant and Non migrant Households within Mexico", International Migration Review, 36 (3), pp. 746-765

Gubert Flore (2005) : " L'impact des transferts de fonds sur le développement des pays d'origine : le cas de l'Afrique ", in OCDE Éd., Migrations, transfert de fonds et développement, Paris, Éditions OCDE, pp. 43-63.

Gubert Flore, Lassourd Thomas et Mesplé-Somps Sandrine (2010) : "Transferts de fonds des migrants, pauvreté et inégalités au Mali. Analyse à partir de trois scénarii contrefactuels ", Revue économique, 6 (I0), pp. I023-1050.

López-Cordova Ernesto (2006): "Globalization, Migration and Development: The Role of Mexican
Migrant Remittances", INTAL-ITD Working Paper 20, 60 p.

Ratha Dilip, Mohapatra et Silwal Ani (20II) : Le Recueil de statistiques 201 I sur les migrations et les envois de fonds, 2 e édition, Banque mondiale, 275 p. [en ligne] consulté le $15 / 01 / 2012$. URL : http://siteresources.worldbank.org/INTPROSPEC TS/

Resources/334934III03I50I5I65/Factbook20IIFrench.pdf.

TAMO M. Eric (20|4) : "Envois de fonds des migrants, pauvreté et inégalités de revenu au Cameroun ", Revue Européenne des Migrations Internationales, 30 (3 \& 4), pp. |8|-200.

Taylor Edward, Mora Jorge, Adams Richard and López-Feldman Alejandro (2008): "Remittances, Inequality and Poverty: Evidence from Rural Mexico", in Migration and Development within and across Borders: Research and Policy Perspectives on Internal and International Migration, Geneva, IOM, pp. I0I-I 28.

Wanner Philippe (2008) : " L'apport des migrants au développement : une perspective économique ", Annuaire suisse de politique de développement, 27 (2), pp. |2|-|3|.

Yang Dean (2008): "International Migration, Remittances and Household Investment Evidence from Philippine Migrants' Exchange Rate Shocks", Economic Journal, I I 8 (528), pp. 59I-630.

Annexe I : Probabilité d'une non-participation du ménage à l'émigration

\begin{tabular}{|c|c|c|c|c|c|c|c|c|}
\hline \multirow{2}{*}{$\begin{array}{l}\text { Type d'émigration } \\
\text { Caractéristique }\end{array}$} & \multicolumn{4}{|c|}{ Emigration interne } & \multicolumn{4}{|c|}{ Emigration internationale } \\
\hline & \multicolumn{2}{|c|}{ Coefficient } & \multicolumn{2}{|c|}{ Effet marginal } & \multicolumn{2}{|c|}{ Coefficient } & \multicolumn{2}{|c|}{ Effet marginal } \\
\hline \multicolumn{9}{|l|}{ Milieu } \\
\hline Dakar & Réf. & & Réf. & & Réf. & & Réf. & \\
\hline Autre urbain & $-0,70$ & *** & $-0,19$ & $* * *$ & 0,38 & **⿻丷木 & 0,08 & $* *$ \\
\hline Rural & $-0,54$ & $* *$ & $-0,14$ & **⿻丷木大米 & $-0,34$ & $*$ & $-0,09$ & $*$ \\
\hline \multicolumn{9}{|l|}{ Ethnie } \\
\hline Bambara & Réf. & & Réf. & & Réf. & & Réf. & \\
\hline Diola & $-1,65$ & **** & $-0,51$ & *米米 & 0,60 & & 0,14 & \\
\hline Pular & $-0,33$ & & $-0,07$ & & 0,07 & & 0,02 & \\
\hline Sérer & $-0,7 \mid$ & & $-0,18$ & $*$ & 0,63 & & 0,14 & \\
\hline Woloff & $-0,65$ & & $-0,16$ & * & 0,15 & & 0,04 & \\
\hline Autre & $-0,59$ & & $-0,14$ & & 0,09 & & 0,02 & \\
\hline Score d'actifs possédés (Log) & 0,48 & $* * *$ & 0,14 & **** & $-0,84$ & $* * *$ & $-0,21$ & $* * *$ \\
\hline Taille du ménage (Log) & $-0,06$ & & $-0,02$ & & $-0,34$ & **** & $-0,09$ & $* * *$ \\
\hline Nombre d'instruits (Log) & $-0,03$ & & $-0,01$ & & $-0,08$ & & $-0,02$ & \\
\hline $\begin{array}{l}\text { Sexe du Chef de ménage } \\
\qquad(0=\text { Homme, I = Femme })\end{array}$ & $-0,28$ & $*$ & $-0,09$ & * & $-0,54$ & $* * *$ & $-0,15$ & $* * *$ \\
\hline Age du Chef de ménage & $-0,03$ & & $-0,01$ & & 0,02 & & 0,01 & \\
\hline Age du chef de ménage au carré & 0,00 & & 0,00 & & 0,00 & & 0,00 & \\
\hline $\begin{array}{l}\text { Situation d'activité du CM } \\
\quad(0=\text { Non occupé, I = Occupé })\end{array}$ & 0,06 & & 0,02 & & 0,15 & & 0,04 & \\
\hline
\end{tabular}




\begin{tabular}{|c|c|c|c|c|}
\hline \multirow{2}{*}{$\begin{array}{l}\text { Type d'émigration } \\
\text { Caractéristique }\end{array}$} & \multicolumn{2}{|c|}{ Emigration interne } & \multicolumn{2}{|c|}{ Emigration internationale } \\
\hline & Coefficient & Effet marginal & Coefficient & Effet marginal \\
\hline Constance & $2,21 \quad * *$ & & $|, 9|$ *** & \\
\hline $\begin{array}{l}\text { Log }=\text { Logarithme } \\
* * * * 0,01, * * * 00,05,{ }^{*} p<0,1\end{array}$ & & & & \\
\hline
\end{tabular}

Source : Calculs des auteurs à partir des données CRES 2009

$\overline{{ }^{\mathrm{i}} \text { Des espoirs déçus et exacerbés entre autres par des crises }}$ économiques et politiques.

${ }^{\text {ii }}$ La méthodologie exposée ici est celle utilisée par Tamo (2014), inspirée de Barham et Boucher (1988).

${ }^{\text {iii }}$ Le choix des variables, ici, est loin d'être un exercice facile, car celles-ci doivent, tout en n'ayant aucun effet sur le revenu du ménage ainsi que les caractéristiques non observables contenues dans le terme d'erreur de l'équation de revenu, influer sur la propension du ménage à participer à l'émigration. La solution couramment utilisée consiste à recourir à des mesures de composition ethnique ou de concentration géographique, dans la mesure où la migration est souvent un phénomène lié à des réseaux et à des pratiques ethniques (Gubert et al., 2010 ; Adams et al., 2008 ; Lachaud, 1999).

${ }^{\text {iv }}$ Données du site http://go.worldbank.org/JITC7NYTT0, 2016 